\title{
Authors Index
}

Agil, I. 943

Alanko, S. 1247

Alharkan, A. 943

Alhendi, H. 943

Allwörden, H. N. von 597

Alnaghmoosh, A. 943

Ambroise, J. P. 421

Andolfatto, M. 167

Andresen, U. 197, 1279

Anttila, R. 1247

Aoki, K. 582

Asaji, T. 611

Babović, V. 1024

Bachler, V. 1027

Baczyński, A. 1009

Baier, G. 1458

Balaban, A. T. 863

Baluja, S. 377

Baran, E. J. 307, 779

Baran, J. 35

Baranowski, B. 565

Barraza, R. 406

Bartosz, G. 1381

Baumann, M. 901

Baumann, W. 395

Beau, W. 310

Becker, P. 849

Becker, R. 511, 1309

Bellisent, M. C. 421

Bellisent, R. 421

Berk, H. L. 1208

Bilal, B. A. 894,901

Bittner, E. R. 1415

Blinc, R. 305

Bobkowski, R. 559

Boddenberg, B. 272, 948

Böhm, M. C. $\quad 670,1346$

Bojarski, C. 1451

Borchers, D. 739

Borchert, G. L. 781

Borschel, E. M. 187, 406

Bosse, H. 1381

Bossmann, E. 426

Boucher, D. 97

Bourham, M. A. 120

Braun, M. 1037, 139

Bremer, F. J. 1327

Brittinger, C. 395

Brockner, W. 511, 513, 515, 517, 593, 1309

Brodersen, H. 935

Brucker, R. 521

Bruhn, B. 333

Brunner, H. 1456

Brunvoll, J. 722, 863

Buback, M. $187,267,406$

Budek, A. M. 889

Budtz-Jørgensen, C. 786

Bühler, E. 7, 421, 507

Büscher, R. 67
Burie, J. 846

Burkert, P. K. 275

Cabus, S. 1321

Caldas, I. L. 1124

Canobbio, E. 1067

Christ, G. 1381

Cioslowski, J. 438, 863

Cox, A. P. 957

Cremers, J. 797

Croci, R. 1067

Cyvin, B. N. 181, 722, 863

Cyvin, S. N. $181,722,863$

Dabrowski, R. 35

Dandapat, B. S. 13

DeDier, B. 994

Deeskow, P. 1181

Deligeorgiev, T. G. 43

Dell, M. P. 1175

Demaison, J. 97, 846, 1241

Detzer, N. 395

Diegruber, H. 970

Dolinšek, J. 305

Doose, J. 705

Dormann, E. 413

Dorset, D. L. 381

Dransfeld, P. 471

Dreizler, H. 72, 79, 83,

197, 207, 392, 495, 501,

957, 1043, 1279, 1402

Dryanska, V. 987

Düchs, D. F. 987

Dygdała, R. S. 559

Ebeling, W. 136

Eisenmann, B. 1309

Elsässer, K. 1181

Endoh, A. 700

Englert, B.-G. 825

Fabian, W. 641

Febbraro, S. 258

Feulner, P. 1333

Finck von Finckenstein, K. Graf 1133

Fleck, S. 57, 645

Fleischhauer, J. 153, 352 . 361, 1027

Fliege, E. 72

Försterling, H. D. 963

Fornili, S. L. 227

Franz, W. 1385

Frees, W. 310

Friesel, M. 565

Fritsch, R. H. 1456

Fröhling, J.-C. 395

Fuchs, A. 319

Fujii, Y. 709

Fukamichi, K. 582

Furukawa, Y. 1410
Gadhi, J. 1241

Gaede, J. 341

Gärtner, H. 1381

Gaur, K. 1257

Geisen, H. 519

Gerads, H. 1327

Gerber, S. 753

Getoff, N. 490

Ghosh, D. 835

Gillette, V. H. 791

Gillies, G. T. 663

Gledhill, I. M. A. 1175

Göbel, K. 431

Gonzáles-Baró, A. C. 779

Gorenflo, R. 1141

Gräff, P. 1079

Granada, J. R. 791

Greening, G. G. W. 1283

Grimm, H. 603

Gripp, J. 392, 1279

Grosse, R. 272

Gryczyński, Z. 617, 808, 1396

Grzeszczak, A. 1372

Grzywacz, J. 123

Guarnieri, A. $\quad 705,1275$

Gutmann, I. 181, 438 ,

$722,863,899,1024$

Gupta, S. 577

Habasaki, J. 377, 683, 695, 1021

Härle, J. 113

Hambsch, F.-J. 786

Hansen, P. G. 781

Haupt, H. 925

Hausser, K. H. 1456

Hawlicka, E. 1014,1305

Heil, M. 543

Heineking, N. 83, 207, 392

Heinzinger, K. 29, 227

Hellberg, M. A. 1175

Hensen, K. 341

Heppke, G. 279

Hermann, E. 1041

Herrmann, W. 922

Herrnegger, F. 1085

Herzel, H. 136

Hess, S. 231

Heyne, E. 352, 1027

Hillig II., K. W. 1415

Hilpert, K. 1327

Hlavacek, V. 994

Hopf, H. 1041

Hora, H. 1239

Horikawa, K. 1410

Horton, W. 1199

Hosoe, M. 606, 709

Huber, H. 753

Huebener, R. P. 441

Hübler, A. 797, 803
Ibrahim, I. H. 444, 774

Igarashi, K. $690,777,853$, $1005,1418,1421$

Itoh, S. 858

Iwadate, Y. 853

Jacob, J. 735

Jäger, H. 429

Jäger, W. 501, 1405

Janssen, R. 994

Jarjis, H. M. 174, 297, 761

Jokić, S. 1024

Jonson, B. 781

Julsrud, S. 463

Jung, W. I. 1037, 1391

Just, K. 236, 241

Kahlert, C. 143, 324

Kakihana, H. 606

Kakihana, M. 477

Kamiński, J. 813

Kasten, W. 79

Kawski. A. $\quad 617,808,813,1396$

Keller, H. J. 603

Kerner, W. 1101, 1154

Khalil, S. M. 174, 297, 761

Khan, Z. H. 91

Kiermeier, A. 1399

Kirste, B. 1296

Kischkel, C. S. 1037

Kiss'ovski, Zh. 1443

Klasinc, L. 293

Klein, M. 655

Klemm, A. 899

Kleppa, O. J. 463

Klessinger, M. 293

Klobasa, D. G. 275

Knitter, H.-H. 786

Knop, J. V. 863

Knopf, K. 909

Kobertz, D. 1327

Koch, W. 875

Kohne, B. 631

Komorowski, L. 767

Koniński, M. 451

Koo, H.-M. 231

Korpiun, P. 922

Kovač, B. 293

Kovačević, M. 863

Kratzsch, K. A. 1225

Krishnan, M. S. 907

Krümpelmann, T. 519

Krzystek, J. 622

Kucinski, M. Y. 1124

Kuczkowski, R. L. 1415

Kübler, J. 1321

Kühner, W. 127

Kume, Y. 611

Lal, H. B. 1257

Lamparter, P. 7, 421, 507, 1385 
Landrath, K. D. 572

Langen, H. 1266

Latowski, T. 1290

Lee, M. L. 735

Legler, W. 935

Legrand, J. 1241

Lehmann, G. 67, 572

Leszcyński, J. 160

Lipiński, J. 160

Lisicki, E. 559

Lochte-Holtgreven, W. 538

Lötzsch, D. 279

Lotz, W. 1045

Lubitz, W. 970

Lüscher, E. 803

Lundén, A. 565, 683

Lutz. O. 1037, 1391

Mäder, H. 501, 1405

Maes, H. 97

Maier, S. 1261

Majerník, V. 1370

Marciniak, B. 485

Martinache, L. 846

Masoud, M. M. 120

Massalski, A. K. 381

Masumoto, T. 582

Matos, J. 1415

Matsubara, E. 582

Matsumoto, A. 447

Mauser, H. 713

Mayer, J. 113

Mayer, K. M. 329,841

Mayer, R. E. 791

McCauly, J. L. 547

Meier, P. 127

Menzel, D. 1333

Merke, I. 495, 846, 1043, 1279

Metropoulos, A. 731

Metzler, W. 310

Meyer, M. 197, 1279

Meyer-ter-Vehn, J. 1096

Migliore, M. 227

Miller, M. 1327

Milstein, F. 889

Mima, K. 1199

Minchev, St. 289

Mizeraczyk, J. 587

Mochinaga, J. $\quad 690,777$ $853,1005,1418,1421$

Möseler, R. 970

Montjoie, A.-S. 1266

Mori, Y. 611

Morrison, P. J. 1115

Morsi, H. W. 1051

Mourad, A. E. 284

Mühlbach, A. 441

Müller, A. 7, 421

Müller, M. 426

Müller, W. R. 863

Müller-Warmuth, W. 1266

Mulser, P. 1147

Nagumo, T. 477,606

Nakamura, D. 611; 1410
Nalewajski, R. F. 451

Narramore, P. 236

Neger, T. 429

Nesper, R. 670

Neue, G. 948

Neuling, H. W. 631

Neuschäfer, D. 519

Neusser, H. J. 1399

Neustock, W. 705

Nickel, H. 1327

Nikolov, P. 43, 289, 490, 987

Noack, F. 127

Nomura, M. 700

Nührenberg, J. 1045

Nugayev, R. M. 657

Odawara, O. 1021

Oeschey, R. 1037, 1391

Oestreicher, F. 279

Ogita, N. 29

Ohtaki, H. 29, 367, 858

Ohtani, H. 1421

Ohzora, Y. 582

Oi, T. 606

Okada, I. 21, 29, 367, 377, $683,695,700,1017,1021$

Okamoto, M. 700, 709

Okamoto, Y. 1005

Osiander, R. 922

Ottinger, Ch. 519

Pachl, R. 963

Pätzmann, U. 513, 515, 517,593

Pajak, Z. 253

Pálinkás, G. 29

Papadopoulos, M. G. 749

Pappas, S. P. 1425

Parisi, J. 263, 329, 441, 655,841

Paso, R. 1247

Peinke, J. 263, 329, 441, 655,841

Perić, M. 103

Pfeffer, M. 1037

Pikuth, A. 935

Pittermann, U. 1366

Polster, J. 636

Port, H. 1261

Praefcke, K. 631

Preetz, W. 597

Probst, M. M. 871

Przedmojski, J. 35

Raabe, G. $\quad 153,352,1027$

Radić-Perić, J. 103

Rakshit. A. K. 903

Ramírez, R. $\quad 670,1346$

Randell, J. 957

Rau, U. 655

Ravn, H. L. 781

Rebhan, E. 794, 1225

Reiser, G. 803

Richter, J. 426

Rode, B. M. 871

Röhr. H. 1051

Röhricht, B. 263, 329, 655, 841
Rössler, O. E. 263, 324, 655

Rommel, E. 127

Rompay, P. van 994

Rose, T. 781

Roy, J. 835

Ruangpornvisuti, V. W. 871

Sahyouni, W. 1443

Sanctuary, B. C. 907

Sasane, A. 611

Saudy, A. H. 120

Schamel, H. 1167

Schlag. E. W. 1399

Schleker, W. 352, 361, 1027

Schlüter, A. 1045

Schmalz, R. 1096

Schmidt, P. C. 1321

Schnabel, W. 1425

Schnering, H. G. von 670

Schreiber. H. 963

Schütz, J. U. von 622

Schulmeister, Th. 136

Schultes, H. 413

Schumacher, U. 1051

Schwager, B. 395

Schwarz, R. 1279

Schweer, J. 267

Schweitzer, D. 603

Seelig, F. F. 875

Sengupta, R. 835

Sharkawy, W. 120

Sharma, S. 1313

Shen. S. 1333

Sheridan, J. 501

Shinohara, H. 611

Siebert, D. 258

Singh, R. A. 577

Sitte, W. 1

Sofroniev, N. 289

Sorg, M. 521

Sosnowska, B. 35

Speake, C. C. 663

Spieckermann, J. 167

Spohr, E. 29, 227

Srzić, D. 293

Stahl, W. 392, 1279, 1402

Steeb, S. 7, 113, 421, 507 1385

Steeb, W.-H. 819

Stegemeyer, H. 631

Stephan, R. 1381

Stezowski, J. J. 622

Stigler, R.-D. 622

Stiller, H. 1266

Stolze, M. 49

Strassburger, W. 1027

Strohriegl, P. 413

Stumpf, H. 213

Sutter, D. H. 49,167

Swietlik, R. 603

Szafrańska, B. 253

Szudy, J. 559

Szymanski, K. 863

Tajima, T. 1199

Tamura, Y. 29, 367

Tanaka, K. 29
Targowski, P. 1009

Tasso, H. 1237, 1377

Taubmann, G. 87

The, L. S. 241

Theobald, J. P. 786

Timtcheva, I. 289, 490

Tonder, A. J. van 819

Tošić, R. 863

Trautwein, A. X. 211

Trinajstić, N. 863

Trumpakaj, Z. 123

Tups, H. 267

Tyutyulkov, N. 43,987

Ueberla, A. 310

Umbach, E. 1333

Urban, K. 113

Vázquez, L. 905

Venugopal, V. 1327

Vojta, G. 556

Vormann, K. 1279

Wagner, H. Gg. 471

Wahl, F. 431

Waite, J. 749

Wallraff, P. 246

Walls, C. T. 501

Walraven, F. 994

Waschkowski, W. 909

Waseda, Y. 582

Waser, R. 1357

Wegmann, K. 1458

Weiden, N. 1313

Weil, K. G. 1283

Weiss, Al. 57, 645, 739, 1313,1321

Weitzner, H. 1101

Wenchen, He. 863

Wenjie, He. 863

Weppner, W. 1

Werheit, H. 925

Wessely, B. 841

Wiczk, W. M. 1290

Winkler, H. 211

Winnewisser, G. 246

Winscom, C. J. 970

Winterberg, F. 1374, 1428

Witalis, E. A. 917

Wlodarczak, G. 97, 1241

Wobig, H. 1054

Wolf, H. C. 622

Wong, H. V. 1208

Wurth, W. 1333

Yadava, Y. P. 577

Yagci, Y. 1425

Yamada, K. M. T. 246

Yamaguchi, T. 367

Yang, Ch. 695, 1017, 1021

Yap, C. T. 1253

Zander, M. 735, 1041

Zechner, J. 490

Zerbe, H. 1275

Zhelyazkov, I. 1443

Ziętek, B, 1009

Zurkowska, G. 1451

Zylka, Ch. 556 